\title{
On Muscle CCO Intermittent Hypoxic Training
}

\author{
Qiao zhen Yan ${ }^{1 . a}$, Ping Wang ${ }^{2 . b}$ and Yong bin Zhao ${ }^{3 . c}$ \\ ${ }^{1.2 .3}$ Department of Physical Education,Wen Shan University, Wen Shan, 663000, China \\ Ayanqiaozhen07212163.coml, 313834775@qq.com, '1093580197@qq.com
}

Keywords: Intermittent Hypoxic Training; skeletal muscle; cytochrome oxidase

\begin{abstract}
Intermittent hypoxic training is one of the more common studies simulated altitude training method, one can accept the hypoxic stimulus, on the other hand to avoid increasing the load on the plateau is not a problem. In this study, changes in 72 male SD rat skeletal muscle cytochrome oxidase in the intermittent hypoxia condition, to investigate the effect of intermittent hypoxic training on aerobic capacity of skeletal muscle in rats.
\end{abstract}

\section{Introduction}

Since the Mexico City Olympics, international sports scientific community set off a wave of altitude training in research, through decades of studies have found that high altitude training pros and cons. So the eyes of scholars turned to either exploit the plateau hypoxia, and also avoid simulated altitude training is difficult to increase the load on the plateau, the HiLo training combination of both to explore the potential of long-Jan altitude training, and also avoid difficult to increase the load on the plateau of the short training ${ }^{[1,2,3]}$. Such as high pressure and high oxygen houses, hypoxia warehouse (hypobaricchamber) and hypoxic tent system (hypoxicotent system) et al ${ }^{[4,5]}$. Numerous studies and proved that these three methods have their advantages and disadvantages, their common feature is persistent hypoxia. Russian and American scholars in the study of the process of gradually realize: Hypoxic Training To achieve the desired effect is not dependent on the persistence of hypoxia and hypoxia exposure duration, the key is repeated from low to high, shift from high to low, $1 \sim 2 \mathrm{~h}$ of intermittent hypoxia stimulate more long-lasting than in the low-oxygen environment can be more effectively improve the ability of the body hypoxia ${ }^{[6]}$. Thus, intermittent hypoxic training as a fourth method came into being. intermittent hypoxic training (IHT) is the use of specialized equipment, by reducing the percentage of oxygen concentration in the air to form an artificial hypoxic environment, so that the subject of intermittent hypoxic gas inhalation, reaching similar purpose with altitude training.

\section{Research content and methods}

\subsection{Object of study}

SD male rats were 72, 8 weeks old, according to the experimental animals fed standard score cages, habilitation one week after the experimental animals were randomly divided into four groups, formal training in three weeks, a total of four weeks.

\subsection{Empirical method}

\subsubsection{Experimental groups}

The rats of adaptation training after a week were randomly divided into 4 groups: normoxic control group (C), normoxic training group (S), intermittent hypoxia control group (I),intermittent hypoxic training group (IS), 18 rats in each group.

\subsubsection{Group training arrangement}

The normoxic control group (C): normal eating, drinking freely without any training activities.

Normally the exercise group (S): the rats every day onlyaerobic endurance training.

The intermittent hypoxia group and hypoxia group (I): IHT in the hypoxic chamber, 3 times a week, 1 hours a day (acombination of 5 minutes, 5 minutes of intermittent hypoxia,a total of 6 
combinations). The first week of oxygenconcentration is $14 \%$, after a week of decline, decline of $1 \%$ per week.

The intermittent hypoxic training group (IS): hypoxia group IHT in the hypoxic chamber, 3 times a week, 1 hours a day(a combination of 5 minutes, 5 minutes of intermittent hypoxia, a total of 6 combinations). The first week of oxygen concentration is $14 \%$, after a week of decline,decline of $1 \%$ per day per week, immediately after hypoxia training of aerobic endurance training.

\subsection{Sport mode:}

The exercise group with domestic rodents electric treadmilltraining, to adapt to the peripheral speed of $15 \mathrm{~m} / \mathrm{min}$, every $30 \mathrm{~min}$, so that the animal be familiar with treadmill exercise,starting 1 week after week incremental load, increase thespeed of $5 \mathrm{~m} / \mathrm{min}$ per week, increase the time of $10 \mathrm{~min}$; the last week of the rate of $30 \mathrm{~m} / \mathrm{min}$, the time of $60 \mathrm{~min}$.

\subsection{Experimental materials}

After the end of training program, with $0.4 \%$ sodium pentobarbital anesthetized rats $\mathrm{lml} / 100 \mathrm{~g}$ weight, quickly remove the gastrocnemius muscle and heart, the heartweighed in Libra, and then use the Department of Ophthalmology small shear separation of left and right ventricular, use vernier caliper to measure left ventricularwall thickness, the gastrocnemius muscle and myocardiumin -70 deg.c for refrigerator.

\subsection{Test index}

Cytochrome oxidase (CCO): measured byspectrophotometer

\subsection{Test index steps}

Weigh accurately myocardial and right gastrocnemius muscle after each $200 \mathrm{mg}, 5 \mathrm{ml}$ respectively into the small beaker; pipettes take advance in the refrigerator (4 DEG C)pre cooling of 9\% $1 \mathrm{ml}$ of physiological saline in a beaker,Department of Ophthalmology, small shear soon chopped tissue block (small beaker in ice water). The chopped tissue homogenate then pour into a glass tube, $0.8 \mathrm{ml}$ cold physio-logical saline residue in the beaker tissue fragments,together into homogenate tube were homogenized to enable the organization fully homogenized, made $10 \%$ homogenate. The preparation of homogenates preparedby freeze centrifuge 3000r/min centrifugal 10min, Torikami Kiyo, according to the determination of cytochrome oxidasedetermination of conventional cytochrome oxidase methodafter centrifugation activity.

\subsection{Data processing}

All the data are based on mean and standard deviation $(\mathrm{X} \pm \mathrm{SD})$ to represent the variance for single factor statisticalprocess analysis method, all data in the computer using the statistical software SPSS which is computed, significant difference is $\mathrm{p}<0.05$.

\section{The results of Experiment}

From table 1 we can see that the changes of cytochrome oxidase in skeletal muscle tissue:Intermittent hypoxia model: intermittent hypoxia control group and normal group there was a significant difference $(\mathrm{p}<0.01)$, intermittent hypoxic training group and thenormal group, normoxic training group, there was verysignificant difference $(\mathrm{p}<0.01)$, intermittent hypoxic training group and intermittent hypoxia control group with significant difference $(p<0.05)$, immediately after the exercise of state and three hours after exercise conditionsshowed no significant difference.

Table 1: the effect of two kinds of models of hypoxia oncytochrome oxidase of rat skeletal muscle cells (Company: Kmin-1/mg)

\begin{tabular}{c|cc|c}
\hline grouping; & rest state & IMPEX & $\begin{array}{c}\text { Three hours after } \\
\text { exercise }\end{array}$ \\
\hline C group & $4.35+0.49$ & $5.08+1.22$ & $5.56+0.85$ \\
S group & $5.06+0.28 \# \#$ & $5.45+0.72$ & $5.27+0.59$ \\
I group & $5.32+0.52 \# \#$ & $5.42+1.75$ & $5.50+1.52$ \\
IS group & $5.92+0.59 \# \# @ @$ & $5.53+0.53$ & $5.88+0.81$ \\
\hline
\end{tabular}


Note:

(1) compared to the control group $\mathrm{p}<0.05$ and \# showednormal oxygen, \#\# showed $\mathrm{p}<0.01$;

(2) and exercise group comparison @ showed p<0.05, P $<0.01 @$ @ show;

(3) compared to the control group $\mathrm{p}<0.05$ delta shown withintermittent hypoxia, delta shows $\mathrm{p}<$ 0.01 ;

(4) and intermittent hypoxia training group showed relatively*** $\mathrm{p}<0.05, \mathrm{p}<0.01$ shows;

\section{Analysis}

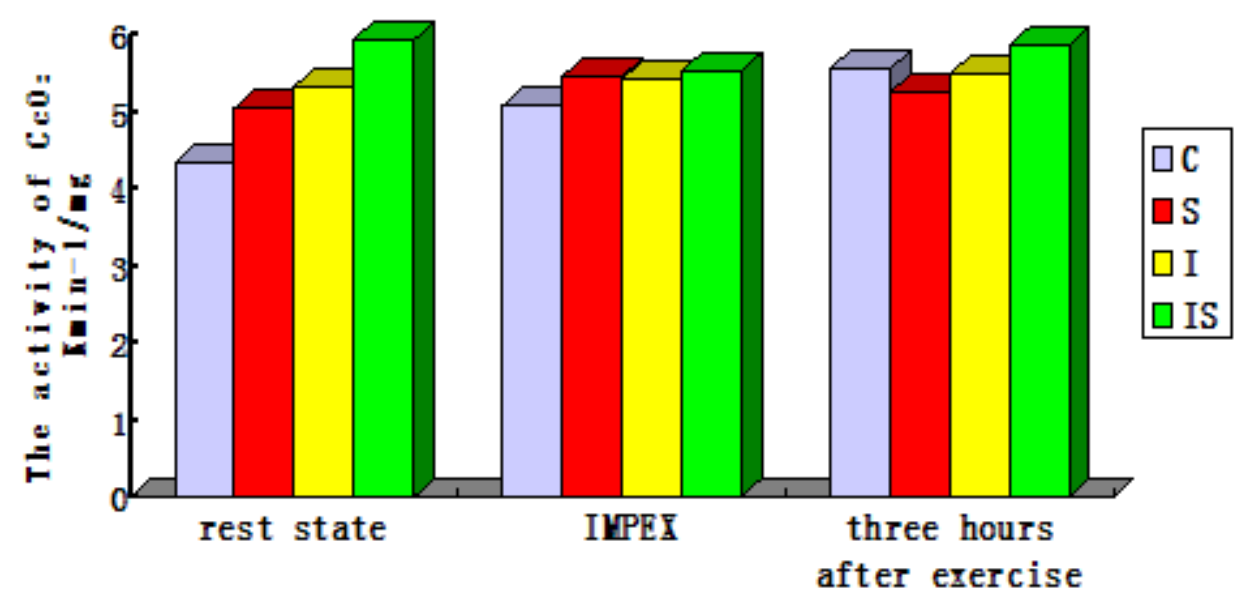

Figure 1: the effect of intermittent hypoxic training onskeletal muscle of rat cytochrome oxidase activity

Mitochondria are cellular respiration and parts produced ATP enzymes, all mitochondrial respiratory chain and oxidative phosphorylation endomembrane containingsystem, including a variety of enzyme NADH dehydrogenase, $\mathrm{CCO}$, electronic through the respiratory chain of these enzymes, and ultimately by the CCO transferto molecular oxygen (components of the electron transport chain through the redox reaction will provide electronicNADH are passed down, the final electron by oxygen molecules to accept, to restore the water, releasing energyfor the body's needs). In these enzymes, cytochromeoxidase (CCO) is a sign of its enzyme. What level of CCO activity and content of it directly affects the efficiency of the respiratory chain electron transfer, determines how muchthe body to produce energy site capacity, maximum working strength will determine the body movement can be reached and longest duration, it directly determines theendurance exercise performance level, which is the most important in the in the movement, the pursue is the entire training target.

Skeletal muscle system function ability and metabolismability height, directly affect the level of the ability ofathletes. Traditional altitude training can have beneficialeffects on the metabolic changes to improve skeletal muscle, such as myoglobin concentration, mitochondrialdensity increased metabolic enzyme activity in muscle was improved in ${ }^{[7,8]}$, intermittent hypoxic training for athletes,can produce similar changes in the same. M.Vogt ${ }^{[9]}$ wasintermittent hypoxia experiments on 30 subjects, subjectstraining every day for 30 minutes, train 5 times a week, a total of 6 weeks. He will be 30 subjects into 4 groups: 2 groups of oxygen partial pressure is 89Torr (1Torr is equivalent to $1 \mathrm{mmHg}$ ) air, simulate the hypoxic environmentat an elevation of $3800 \mathrm{~m}$, one group with high intensity training (training when the lactate concentration is 4 6mmolPL), high intensity in hypoxia group; another group with low intensity training (training of blood lactic acidconcentration is 2 3mmolPL), low intensity in hypoxia group; in the other 2 groups under normoxia training environment, a group with low intensity training, lowintensity normoxic group, another group with high intensity training, high intensity normoxic group. The results show: after intermittent hypoxic training, skeletal musclemitochondria significantly increased overall density with high strength in hypoxia group, mitochondrial densityincreased most, 55\%; low intensity in hypoxia group wholemitochondrial density has increased, only 24.1\%; andtraining under normoxia environment, it has no obviouschange. High intensity in hypoxia group, high intensitynormoxic group and hypoxic 
group of low intensityintermittent hypoxic training, muscle fiber in mitochondrialdensity also increased significantly, respectively $39.3 \%, 2.6 \%, 11.8 \%$, and no obvious change of low intensitynormoxic group. The length and density of capillaries in skeletal muscle only increased significantly in high intensityin hypoxia group, an increase of $18.7 \%$. High strengthtraining in hypoxia group in the intermittent hypoxiatraining, cytochrome oxidase 1 (COX-1) and cytochromeoxidase 4 (COX-4) mRNA concentration significantly increased, an increase of $42.3 \%$ and $45.4 \%$ respectively,NADH $\mathrm{Hu}$ Bo acid dehydrogenase (SDH) mRNAconcentrations also increased significantly, increased to $36.7 \%$ and $46.9 \%$ respectively.

The experimental results indicated that under the quiet state, normoxic control group and exercise group andintermittent hypoxia control group and intermittent hypoxiaexercise group, exercise group and intermittent hypoxia training group with a very significant difference $(p<0.01)$;intermittent hypoxia control group and intermittent hypoxic exercise group were significantly different $(\mathrm{p}<0.05)$,intermittent hypoxia control group increased $22 \%$,intermittent hypoxic training group increased 36\%;immediately after exercise and three hours after exercisecondition, there were no significant differences between the. Description of skeletal muscle cells CCO in quietcondition is quite obvious, but there were no significantchanges in the condition of stress, may need a longer recovery period can have significant differences, due to the limitation of experimental conditions, the experiment failed to explore more deeply.

Findings of this study, skeletal muscle tissue, normoxiccontrol group in a quiet state and the other 5 groups have significant differences in CCO activity, exercise group andintermittent hypoxia training group also has significant differences. However, intermittent hypoxia control groupand intermittent hypoxia training group compared with no significant difference, indicates that the effect of hypoxia in this experiment only applied to hypoxia failed to bodyreaching movements, failed to stimulate skeletal muscle tissue in the CCO to increase its activity, the experimentresults show that, for many IHT in skeletal muscle CCO activity or content has great influence, and the influence ispositive; but three hours immediately after exercise andafter exercise status were not significant difference.IN.Mankovskaya 32 Wsitar rats as the experimental object,to IHT, the equivalent of 8000 $\mathrm{m}$ altitude oxygen partial pressure, train 4 times a day, each time 30min hypoxia, 15-20min interval, a total of 14 days. Experimental study found that, in IHT group than those in normoxic control group CCO in cardiac, liver, brain activity were increased by $40.5 \%, 41.7 \%$ and $20.2 \%$, proved that intermittent hypoxia training enhances the tissue CCO activity, thus stimulating the respiratory chain oxidation pathway, the body's aerobic metabolism ability strengthened, the experimental resultsand the experimental results are similar, on the other handalso support the results of this experiment.

\section{Conclusion}

In this experiment, in a quiet state, normoxic training groupand intermittent hypoxia control group and intermittent hypoxia training group compared to constant activity wasoxygen control of CCO in skeletal muscle of rats wassignificantly increased, and immediately after exercise andthree hours after exercise showed no significant changes.Instructions in a quiet state is CCO in rat skeletal muscleactive state, so the more obvious changes, includingchanges in intermittent hypoxic training group was the most obviously.

\section{References}

[1] Levine BD,tray-Gundersen J:Anat Rec,Vol.3(1997)No.248(3), p.366-373.

[2] Stray-GundersenJ,LevineBD:estnAkadMedNaukSSSR,Vol.5(1988), p.77-80.

[3] Z.P.Lei:Journal of Xi'an Physical Education University,Vol. 2(1997)No.14, p.79-81. “In Chinese".

[4] HellemansJ:A pilot Stuy Mol Cell Cardiol,Vol.6(1996)No.28, p.1271-1277.

[5] Kolchinskaya, Tkatchouk EN:HypMedJ,Vol.1(1993) p.9-18. 
[6] Wilber RL:Sports Med,Vol.4( 2001)No.31, p.249-265.

[7] Terrados N.et al:Int.J Sport Med,Vol.13(1992), p.206-209.

[8] MizunoM.et al:J.Appl, Physiol,Vol.2(1990)No.68, p.496-502.

[9] M.Vogt,A.Puntschart,J.Geiser,C.Zuleger,R.Billete,andH.Hoppeler:J.Appl Physiol,2001,No.91 p.171-182.

[10] P.Liu and Y.Hu:Journal of Shandong Institute of Physical Education and Sports,Vol.4(2004)No.20:65-67.“In Chinese”. 